International Journal of Pure and Applied Mathematics

Volume $90 \quad$ No. 2 2014, 239-254

ISSN: 1311-8080 (printed version); ISSN: 1314-3395 (on-line version)

url: http://www.ijpam.eu

doi: http://dx.doi.org/10.12732/ijpam.v90i2.11

ijpam.eu

\title{
DISCONTINUOUS OBLIQUE DERIVATIVE PROBLEM FOR NONLINEAR ELLIPTIC SYSTEM OF SECOND ORDER EQUATIONS IN MULTIPLY CONNECTED DOMAINS
}

\author{
GuoChun Wen ${ }^{1}$, Yanhui Zhang ${ }^{2}$ \\ ${ }^{1}$ LMAM, School of Mathematical Sciences \\ Peking University \\ Beijing, 100871, P.R. CHINA \\ ${ }^{2}$ Department of Mathematics \\ Beijing Technology and Business University \\ Beijing, 100048, P.R. CHINA
}

\begin{abstract}
In this article, we discuss that the discontinuous oblique derivative boundary value problem for nonlinear uniformly elliptic system of second order equations in multiply connected domains. We first propose the discontinuous oblique derivative problem and its new modified well-posedness. Next we give a priori estimates of solutions of the modified discontinuous boundary value problem for corresponding elliptic system of first order complex equations and verify its solvability by the above estimates of solutions and the Leray-Schauder theorem. Finally the solvability results of the original discontinuous oblique derivative problem can be derived. Here we mention the discontinuous boundary value problems possess many applications in mechanics and physics etc.
\end{abstract}

AMS Subject Classification: 35J57, 35J60, 35B45

Received: October 26, 2013

(C) 2014 Academic Publications, Ltd. url: www.acadpubl.eu

${ }^{\S}$ Correspondence author 


\section{Formulation of Oblique Derivative Problems for Nonlinear Elliptic System of Second Order Equations}

In [1-5], the authors discussed some continuous boundary value problems for elliptic equations and their applications, and in $[4,6]$, some discontinuous boundary value problems for spacial elliptic complex equations were discussed. In this article we shall consider the discontinuous oblique derivative problem for general nonlinear elliptic system of second order equations in multiply connected domains. We first introduce the nonlinear uniformly elliptic systems of second order equations

$$
\begin{aligned}
& u_{j z \bar{z}}=F_{j}\left(z, u_{1}, u_{2}, u_{1 z}, u_{2 z}, u_{1 z z}, u_{2 z z}\right), F_{j}=\operatorname{Re}\left[Q_{j 1} u_{1 z z}+Q_{j 2} u_{2 z z}\right. \\
& \left.+A_{j 1} u_{1 z}+A_{j 2} u_{2 z}+A_{j 3} u_{1}+A_{j 4} u_{2}+A_{j 5}\right], Q_{j l}=Q_{j l}\left(z, u_{1}, u_{2}, u_{1 z}, u_{2 z},\right. \\
& \left.u_{1 z z}, u_{2 z z}\right), l=1,2, A_{j l}=A_{j l}\left(z, u_{1}, u_{2}, u_{1 z}, u_{2 z}\right), l=1, . ., 5, j=1,2,
\end{aligned}
$$

in an $N+1$-connected domain $G$. Denote by $\Gamma=\cup_{j=0}^{N} \Gamma_{j}$ the boundary contours of the domain $G$ and let $\Gamma \in C_{\mu}^{2}(0<\mu<1)$. Without loss of generality, we assume that $G$ is a circular domain in the unit disc $|z|<1$, bounded by the $(N+1)$-circles $\Gamma_{k}:\left|z-z_{k}\right|=r_{k}, k=0,1, \ldots, N$ and $\Gamma_{0}=\Gamma_{N+1}:|z|=1, z=0 \in$ $G$. In this article, the notations are the same as in References [3-12]. Suppose that the system (1.1) satisfies

Condition C. 1) $Q_{j l}\left(z, u_{1}, u_{2}, u_{1 z} u_{2 z}, U, V\right)(l=1,2)$ and $A_{j l}\left(z, u_{1}, u_{2}, u_{1 z}\right.$, $\left.u_{2 z}\right)(l=1, \ldots, 5, j=1,2)$ are continuous in $u_{1}, u_{2} \in \mathbb{R}$ (the real axis) and $u_{1 z}, u_{2 z} \in \mathbb{C}$ (the complex plane) for almost every $z \in G, U, V \in \mathbb{C}$. In addition, let

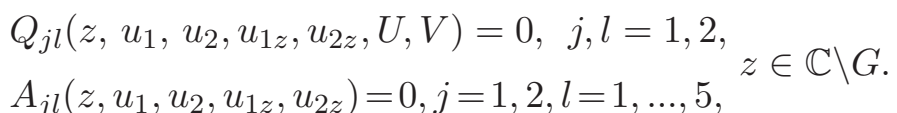

2) The above functions are measurable in $z \in G$ for all continuously differentiable functions $u_{1}(z), u_{2}(z)$ in $G^{*}=\bar{G} \backslash Z$ and any measurable function $U(z), V(Z) \in G^{*}$, and satisfy

$$
\left.L_{p}\left[A_{j l}, \bar{G}\right)\right] \leq k_{j l}<k_{0}, j=1,2, l=1, \ldots, 5,
$$

in which $G^{*}=\bar{G} \backslash Z, Z=\left\{t_{1}, \ldots, t_{m}\right\}, t_{j}(j=1, \ldots, m)$ are distinct points on $\Gamma$, and $p_{0}, p\left(2<p_{0}<p\right), k_{j l}(j=1,2, l=1, \ldots, 5),, k_{0}$ are all positive constants.

3) The system (1.1) satisfies the uniform ellipticity condition, i.e.

$$
\begin{gathered}
\left|F_{j}\left(z, u_{1}, u_{2}, u_{1 z} u_{2 z}, U_{1}, V_{1}\right)-F_{j}\left(z, u_{1}, u_{2}, u_{1 z} u_{2 z}, U_{2}, V_{2}\right)\right| \\
\leq q_{j 0}\left|U_{1}-U_{2}\right|+q_{j 0}^{\prime}\left|V_{1}-V_{2}\right|
\end{gathered}
$$


for arbitrary continuously differentiable functions $u_{1}(z), u_{2}(z)$ in $G^{*}$, almost every point $z \in G$ and $U_{1}, U_{2} \in \mathbb{C}$, where

$$
q_{j 0}, q_{j 0}^{\prime}\left(q_{j 0}+q_{j 0}^{\prime}<1, j=1,2\right)
$$

are non-negative constants.

Problem P. The discontinuous oblique derivative boundary value problems for the system (1.1) may be formulated as follows: To find a system of continuously differentiable solutions $\left(u_{1}(z), u_{2}(z)\right)$ in $G^{*}$ satisfying the boundary conditions

$$
\frac{\partial u_{j}}{\partial v_{j}}+2 \sigma_{j 1}(t) u_{1}(t)+2 \sigma_{j 2}(t) u_{2}(t)=2 \tau_{j 3}(t), t \in \Gamma^{*}=\Gamma \backslash Z, j=1,2,
$$

in which $v_{j}(j=1,2)$ are the vectors on $\Gamma$. Since

$$
\frac{\partial u_{j}}{\partial v_{j}}=\frac{\partial u_{j}}{\partial x} \cos \alpha_{j 1}+\frac{\partial u_{j}}{\partial y} \cos \alpha_{j 2}=2 \operatorname{Re}\left[\overline{\lambda_{j}(t)} u_{j t}\right], \overline{\lambda_{j}(t)}=\cos \alpha_{j 1}+i \cos \alpha_{j 2},
$$

where $\cos \alpha_{j 1}=\cos \left(v_{j}, x\right), \cos \alpha_{j 1}=\cos \left(v_{j}, y\right)$, hence (1.5) can be written in the form

$$
\operatorname{Re}\left[\overline{\lambda_{j}(t)} u_{j t}\right]+\sigma_{j 1}(t) u_{1}(t)+\sigma_{j 2}(t) u_{2}(t)=\tau_{j 3}(t), t \in \Gamma^{*}, j=1,2 .
$$

It is clear that $\left|\lambda_{j}(t)\right|=1(j=1,2)$. In (1.5) and (1.7), $Z=\left\{t_{1}, t_{2}, \ldots, t_{m}\right\}$ is the set of first kind discontinuous points of $\lambda_{j}(z)(j=1,2)$ on $\Gamma, \hat{\Gamma}_{l}$ is an arc from the point $t_{l-1}$ to $t_{l}$ on $\hat{\Gamma}, \hat{\Gamma}_{l}(l=1,2, \ldots, m)$ does not include end points. We can assume that $t_{l} \in \Gamma_{0}\left(l=1, \ldots, m_{0}\right), t_{l} \in \Gamma_{1}\left(l=m_{0}+1, \ldots, m_{1}\right), \ldots, t_{l} \in$ $\Gamma_{N}\left(l=m_{N-1}+1 \ldots, m\right)$ are all discontinuous points of $\lambda_{j}(z)$ on $\Gamma$. Denote by $\lambda_{j}\left(t_{l}-0\right)$ and $\lambda_{j}\left(t_{l}+0\right)$ the left limit and right limit of $\lambda_{j}(z)$ as $z \rightarrow t_{l}(\in \Gamma, l=$ $1,2, \ldots, m, j=1,2)$, and

$$
\begin{aligned}
\mathrm{e}^{\mathrm{i} \phi_{j l}} & =\frac{\lambda_{j}\left(t_{l}-0\right)}{\lambda_{j}\left(t_{l}+0\right)}, \gamma_{j l}=\frac{1}{\pi \mathrm{i}} \ln \left[\frac{\lambda_{j}\left(t_{l}-0\right)}{\lambda_{j}\left(t_{l}+0\right)}\right]=\frac{\phi_{j l}}{\pi}-K_{j l}, \\
K_{j l} & =\left[\frac{\phi_{j l}}{\pi}\right]+J_{j l}, J_{j l}=0 \text { or } 1, j=1,2, l=1, \ldots, m,
\end{aligned}
$$

in which $0 \leq \gamma_{j l}<1$ when $J_{j l}=0$, and $-1<\gamma_{j l}<0$ when $J_{j l}=1, j=$ $1,2, l=1, \ldots, m$. There is no harm in assuming that the partial indexes $\tilde{K}_{j l}=$ $\sum_{j=m_{l-1}+1}^{m_{l}} K_{j l}$ are denoted the partial indexes of $\lambda_{j}(z)$ on $\Gamma_{l}(l=0,1, \ldots, N)$, and the partial indexes $\tilde{K}_{j l}$ on $\Gamma_{l}\left(l=1, \ldots, N_{0}(\leq N)\right)$ are not integers and $\tilde{K}_{j l}=\sum_{j=m_{l-1}+1}^{m_{l}} K_{j l}$ of $\lambda_{j}(z)$ on $\Gamma_{l}\left(l=N_{0}+1, \ldots, N\right)$ are integers. Set

$$
K_{j}=\frac{1}{2 \pi} \Delta_{\Gamma} \arg \lambda_{j}(z)=\sum_{l=1}^{m} \frac{K_{j l}}{2}, j=1,2
$$


and $K=\left(K_{1}, K_{2}\right)$ is called the index of Problem P. Moreover, $\lambda_{j}(z), r_{j}(z)$ $(j=1,2)$ satisfies the conditions

$$
\begin{gathered}
C_{\alpha}\left[\lambda_{j}(z), \hat{\Gamma}_{l}\right] \leq s_{0}, C_{\alpha}\left[\left|z-t_{l}\right|^{\beta_{j l}}\left|z-t_{l-1}\right|^{\beta_{j l-1}} \sigma_{j n}, \hat{\Gamma}_{l}\right] \leq s_{1}, j, n=1,2, \\
C_{\alpha}\left[\left|z-t_{l}\right|^{\beta_{j l}}\left|z-t_{l-1}\right|^{\beta_{j l-1}} \tau_{j 3}(z), \hat{\Gamma}_{l}\right] \leq s_{0}, j=1,2, l=1, \ldots, m,
\end{gathered}
$$

in which $s_{0}, s_{1}\left(s_{1} \leq s_{0}\right), \alpha(1 / 2<\alpha<1)$ are non-negative constants, $\beta_{j l}+\left|\gamma_{j l}\right|<$ $1, j=1,2, l=1, \ldots, m$; we require that the solution $\left[w_{1}(z), w_{2}(z)\right]$ possesses the property

$$
\begin{aligned}
& R(z) w_{j}(z)=C_{\delta}(\bar{G}), R(z)=\Pi_{l=1}^{m}\left|z-t_{l}\right|^{\eta_{l} / \tau^{2}}, \eta_{l}=\max \left(\eta_{1 l}, \eta_{j 2}\right), \\
& \eta_{j l}=\left\{\begin{array}{l}
\beta_{j l}+\tau, \text { for } \gamma_{j l} \geq 0, \text { and } \gamma_{j l}<0, \beta_{j l} \geq\left|\gamma_{j l}\right|, \quad j=1,2 \\
\left|\gamma_{j l}\right|+\tau, \text { for } \gamma_{j l}<0, \beta_{j l}<\left|\gamma_{j l}\right|, l=1, \ldots, m,
\end{array}\right.
\end{aligned}
$$

in the neighborhood $(\subset G)$ of $t_{l}(l=1, \ldots, m)$, where $\delta, \tau\left(\leq \min \left(\alpha, 1-2 / p_{0}\right)\right)$ are small positive constants.

We mention that the first boundary value problem, second boundary value problem and third boundary value problem, i.e. regular oblique derivative problem are the special cases of Problem P, because their boundary conditions are the continuous boundary conditions, and their indexes are equal to $K_{j}=N-1(j=1,2)$. Now $2 K_{j}(j=1,2)$ can be any positive or negative integers, hence Problem $\mathrm{P}$ is a vary general discontinuous boundary value problem.

In the next section on we first introduce the modified boundary value problems (Problem Q) for elliptic system of first order complex equations, which corresponds to Problem $\mathrm{P}$ for the system (1.1). Moreover we establish then the integral expression and a priori estimates of solutions for Problem Q. By the estimates of solutions and the Leray-Schauder theorem, we can prove the existence of solutions for Problem Q, and so derive the results of the solvability for Problem $\mathrm{P}$ for the system (1.1) with some conditions as follows.

Theorem 1.1. (The Main Theorem) Suppose that the second order nonlinear system (1.1) satisfy Condition C. If the constants $q_{10}^{\prime}, k_{1 l}(l=2,3,4), k_{24}$ in (1.3), (1.4) and $s_{1}$ in (1.10) are all sufficiently small, then Problem $\mathrm{P}$ for (1.1) possesses the following results on solvability:

(1) When the indexes $K_{j}=\frac{1}{2 \pi} \Delta_{\Gamma} \arg \lambda_{j}(t) \geq 0(j=1,2)$, Problem $\mathrm{P}$ for (1.1) has $4 N$ solvability conditions, and the solution depends on $2\left(K_{1}+K_{2}+2\right)$ arbitrarily real constants.

(2) When $K_{1}<0, K_{2}<0$, Problem P has $4 N-2 K_{1}-2 K_{2}-2$ solvability conditions, and the solution depends on two arbitrarily real constants. 
Moreover, we can write down the solvability conditions of Problem P for all other cases.

For Problem P in the simply connected domain, we considered its solvability, the result obtained is a special case of Theorem 1.1, which can be seen from Chapter VI, [3].

\section{Formulation of Modified Problem for Nonlinear Elliptic System of First Order Equations}

Now we introduce the new well posedness of discontinuous oblique derivative problem for the elliptic system (1.1), namely

Problem Q. To find a system of continuous solutions $\left(u_{1}(z), u_{2}(z), w_{1}(z)\right.$, $\left.w_{2}(z)\right)$ in $G$ for the nonlinear system of first order equations

$$
\begin{aligned}
& w_{j \bar{z}}=f_{j}\left(z, u_{1}, u_{2}, w_{1}, w_{2}, w_{1 z}, w_{2 z}\right), f_{j}=\operatorname{Re}\left[Q_{j 1} w_{1 z}+Q_{j 2} w_{2 z}\right. \\
& \left.+A_{j 1} w_{1}+A_{j 2} w_{2}+A_{j 3} u_{1}+A_{j 4} u_{2}+A_{j 5}\right], Q_{j l}=Q_{j l}\left(z, u_{1}, u_{2}, w_{1}, w_{2},\right. \\
& \left.w_{1 z}, w_{2 z}\right), j, l=1,2, A_{j l}=A_{j l}\left(z, u_{1}, u_{2}, w_{1}, w_{2}\right), j=1,2, l=1, . ., 5,
\end{aligned}
$$

with the boundary conditions

$$
\begin{gathered}
\operatorname{Re}\left[\overline{\lambda_{j}(t)} w_{j}(t)\right]+\sigma_{j 1}(t) u_{1}(t)+\sigma_{j 2}(t) u_{2}(t)=\tau_{j 3}(t)+h_{j}(t) \overline{\lambda_{j}(z)} X_{j}(z), t \in \Gamma^{*}, \\
j=1,2 .
\end{gathered}
$$

in which

$$
h_{j}(z)=\left\{\begin{array}{l}
h_{j l} z \in \Gamma_{l}, l=1, \ldots, N, \\
0, z \in \Gamma_{0}, z \in \Gamma_{0}, \\
h_{j l}, z \in \Gamma_{l}, l=1, \ldots, N \\
{\left[1+(-1)^{2 K_{j}}\right] h_{0}+\operatorname{Re} \sum_{m=1}^{\left[\mid K_{j}+1 / 2\right]-1}\left(h_{j m}^{+}+\mathrm{i} h_{j m}^{-}\right) z^{m}, z \in \Gamma_{0}}
\end{array}\right\} \text { if } K_{j} \geq 0,
$$


in which $h_{j l}\left(l=\left[1-(-1)^{2 K_{j}}\right] / 2, \ldots, N\right)$ and $h_{j m}^{ \pm}\left(m=1, \ldots,\left[\left|K_{j}\right|+1 / 2\right]-1, j=\right.$ $1,2)$ are unknown real constants to be determined appropriately, and

$$
\begin{gathered}
Y_{j}(z)=\prod_{l=1}^{m_{0}}\left(z-t_{l}\right)^{\gamma_{j l}} \prod_{s=1}^{N}\left(z-z_{s}\right)^{-\left[\tilde{K}_{j s}\right]} \prod_{l=m_{0}+1}^{m_{1}}\left(\frac{z-t_{l}}{z-z_{1}}\right)^{\gamma_{j l}} \cdots \prod_{l=m_{N_{0}-1}+1}^{m_{N_{0}}}\left(\frac{z-t_{l}}{z-z_{N_{0}}}\right)^{\gamma_{j l}} \\
\times \prod_{l=m_{N_{0}+1}}^{m_{N_{0}+1}}\left(\frac{z-t_{l}}{z-z_{N_{0}+1}}\right)^{\gamma_{j l}}\left(\frac{z-t_{N_{0}+1}^{\prime}}{z-z_{N_{0}+1}}\right) \cdots \prod_{l=m_{N-1}+1}^{m}\left(\frac{z-t_{l}}{z-z_{N}}\right)^{\gamma_{j l}}\left(\frac{z-t_{N}^{\prime}}{z-z_{N}}\right), \\
j=1,2,
\end{gathered}
$$

where $\tilde{K}_{j s}=\sum_{l=m_{s-1}+1}^{m_{s}} K_{j l}(j=1,2)$ is denoted the partial indexes on $\Gamma_{s}(s=$ $1, \ldots, N)$; there is in no harm assuming that the partial indexes $K_{j s}$ of $\lambda_{j}(z)$ on $\Gamma_{s}\left(s=0, \ldots, N_{0}(\leq N), j=1,2\right)$ are integers, and the partial indexes $K_{j s}$ of $\lambda_{j}(z)$ on $\Gamma_{s}\left(s=N_{0}+1, \ldots, N, j=1,2\right)$ are no integers, and $t_{l}^{\prime}\left(\in \Gamma_{l}, l=N_{0}+1, \ldots, N\right)$ are fixed points, which are not the discontinuous points at $Z$; we must give the attention that the boundary circles $\Gamma_{l}(l=0,1, \ldots, N)$ of the domain $G$ are moved round the positive direct. Similarly to (1.7)-(1.12), Chapter V, [4], we see that

$$
\frac{\lambda_{j}\left(t_{l}-0\right)}{\lambda_{j}\left(t_{l}+0\right)} \overline{\left[\frac{Y_{j}\left(t_{l}-0\right)}{Y_{j}\left(t_{l}+0\right)}\right]}=\frac{\lambda_{j}\left(t_{l}-0\right)}{\lambda_{j}\left(t_{l}+0\right)} e^{-i \pi \gamma_{j l}}= \pm 1, j=1,2, l=1, \ldots, m,
$$

it only needs to charge the symbol on some arcs on $\Gamma$, then $\lambda_{j}(z) \overline{Y_{j}(z)} /\left|Y_{j}(z)\right|$ $(j=1,2)$ on $\Gamma$ are continuous. In this case, its index

$$
\kappa_{j}=\frac{1}{2 \pi} \Delta_{\Gamma}\left[\lambda_{j}(z) \overline{Y_{j}(z)}\right]=K_{j}-\frac{N-N_{0}}{2}, j=1,2
$$

are integers; and

$$
\begin{aligned}
& X_{j}(z)=\left\{\begin{array}{l}
i z^{\left[\kappa_{j}\right]} e^{i S_{j}(z)} Y_{j}(z), z \in \Gamma_{0}, \\
i e^{i \theta_{j l}} e^{i S_{j}(z)} Y_{j}(z), z \in \Gamma_{l}, l=1, \ldots, N,
\end{array}, \operatorname{Im}\left[\overline{\lambda_{j}(z)} X_{j}(z)\right]=0, z \in \Gamma,\right. \\
& \operatorname{Re} S_{j}(z)=\left\{\begin{array}{l}
\arg \lambda_{j}(z)-\left[\kappa_{j}\right] \arg z-\arg Y_{j}(z), z \in \Gamma_{0}, \\
\arg \lambda_{j}(z)-\arg Y_{j}(z)-\theta_{j l}, z \in \Gamma_{l}, l=1, \ldots, N,
\end{array} \quad \operatorname{Im}\left[S_{j}(1)\right]=0,\right. \\
& j=1,2,
\end{aligned}
$$

in which $S_{j}(z)(j=1,2)$ are solutions of the modified Dirichlet problem with the above boundary conditions for analytic functions. 
Moreover we suppose that the solution satisfies the point conditions:

$$
\operatorname{Im}\left[\overline{\lambda_{j}\left(a_{l}\right)} w_{j}\left(a_{l}\right)\right]=b_{j l}, l \in J_{j}, J_{j}=\left\{1, \ldots, 2 K_{j}+1\right\}, K_{j} \geq 0, j=1,2,
$$

where $a_{l}\left(\in \Gamma_{0}, l \in J_{j}, K_{j} \geq 0, j=1,2\right)$ are distinct points; and $b_{j l}\left(l \in J_{j}, j=1,2\right)$ are all real constants satisfying the conditions

$$
\left|b_{j l}\right| \leq s_{0}<\infty, l \in J_{j}, j=1,2 .
$$

Besides, we require that the solution $\left(u_{1}(z), u_{2}(z), w_{1}(z), w_{2}(z)\right)$ satisfies $R^{\prime}(z)$ $u_{j}(z) \in C_{\alpha}^{1}(\bar{G}), R S w_{j}(z) \in W_{p_{0}}^{1}(G)\left(\alpha=1-2 / p_{0}, 2<p_{0}<p, j=1,2, S(z)=\right.$ $\left.\Pi_{l=1}^{m}\left|z-t_{l}\right|^{l / \tau^{2}}\right)$ and the relation

$$
u_{j}(z)=b_{j 0}+\operatorname{Re} \int_{a_{0}}^{z}\left[w_{j}(z)+\sum_{k=1}^{N} \frac{i d_{j k}}{z-z_{k}} \mathrm{~d} z\right],
$$

where $a_{0}=1,\left|b_{j 0}\right| \leq s_{0}, d_{j k}(j=1,2, k=1, \ldots, N)$ are appropriate real constants such that the functions $u_{j}(z)(j=1,2)$ determined by the integral in (2.6) is single-valued in $G$.

Theorem 2.1. (The Equivalence Theorem) Let $\left(u_{1}(z), u_{2}(z)\right)$ be a solution of Problem $\mathrm{P}$ for the second order system (1.1). Then $\left(u_{1}(z), u_{2}(z)\right.$, $\left.w_{1}(z), w_{2}(z)\right)$ is a solution of Problem Q for the system (2.1) of first order, which satisfies the boundary conditions

$$
\operatorname{Re}\left[\overline{\lambda_{j}(t)} w_{j}(t)\right]+\sigma_{j 1}(t) u_{1}(t)+\sigma_{j 2}(t) u_{2}(t)=\tau_{j 3}(t), t \in \Gamma, j=1,2,
$$

and the relation (2.6). Conversely, let $\left(u_{1}(z), u_{2}(z), w_{1}(z), w_{2}(z)\right)$ be a solution of Problem Q for the system (2.1), which satisfy the boundary conditions (2.2)(2.5) and the relation (2.6) with $h_{j}(t)=0$ and $d_{j k}=0(j=1,2, k=1, \ldots, N)$. Then $\left(u_{1}(z), u_{2}(z)\right)$ is a solution of Problem $\mathrm{P}$ for (1.1).

The proof of the theorem is immediate.

\section{Estimates of Solutions of Modified Problem for Nonlinear Elliptic System of First Order Equations}

In this section, we shall give a priori estimates of solutions for Problem Q.

Theorem 3.1. Let $\left(u_{1}(z), u_{2}(z), w_{1}(z), w_{2}(z)\right)$ be a solution of Problem Q for the first order system (2.1) satisfying the conditions

$$
\begin{gathered}
C\left[R^{\prime}(z) u_{j}(z), \bar{G}\right] \leq M_{1}, C\left[R(z) w_{j}(z), \bar{G}\right], \\
\left\|R S w_{j \bar{z}}\right\|_{L_{p_{0}}(\bar{G})}=\left\|R S \rho_{j}(z)\right\|_{L_{p_{0}}(\bar{G})} \leq M_{2}, j=1,2,
\end{gathered}
$$


where $S(z)=\Pi_{l=1}^{m}\left|z-t_{l}\right|^{l / \tau^{2}}, M_{1}, M_{2}$ are unknown real constants to be determined appropriately. Then when the constants $q_{10}^{\prime}, k_{1 l}(l=2,3,4), k_{24}$ in $(1.3),(1.4)$ and $s_{1}$ in (1.10) are sufficiently small, the solution $\left(u_{1}(z), u_{2}(z), w_{1}(z)\right.$, $\left.w_{2}(z)\right)$ certainly satisfies the estimates

$$
\begin{gathered}
C_{\delta}\left[R^{\prime}(z) u_{j}(z), \bar{G}\right]<M_{3}, C_{\delta}\left[R(z) w_{j}(z), \bar{G}\right]<M_{4}, \\
\left\|R S\left(\left|w_{j z}\right|+\left|w_{j z}\right|\right)\right\|_{L_{p_{0}}(\bar{G})}<M_{5}, j=1,2,
\end{gathered}
$$

in which $M_{k}=M_{k}\left(q_{0}, p_{0}, k_{0}, \delta, s_{0}\right)(k=3,4,5), q_{0}=\max \left(q_{10}, q_{20}^{\prime}\right), \delta$ is a small positive constant, and we may be choose that $M_{1}$ and $M_{2}$ are equal to $M_{3}$ and $M_{4}$ respectively.

Proof. We first consider the case $K_{j} \geq 0(j=1,2)$. Let the stated solution $u_{j}(z), w_{j}(z)(j=1,2)$ in the theorem substitute the corresponding functions in the system (2.1), the boundary conditions (2.2)-(2.5) and the formula (2.6). It is clear that $w_{2}(z)$ is a solution of the modified Riemann-Hilbert boundary value problem for the complex equation $w_{2 \bar{z}}=\rho_{2}(z)$ with the boundary condition $(2.2)-(2.6)(j=2)$. By virtue of the condition (3.1) and using the method analogous to that in Theorem 3.1, Chapter V, [3], we can prove that $w_{2}(z)$ satisfies the estimations

$$
C_{\delta}\left[R(z) w_{2}(z), \bar{G}\right]<M_{6},\left\|R S w_{2 z}\right\|_{L_{0}(\bar{G})} \leq M_{7}
$$

where $\delta$ and $p_{0}$ are similar to those mentioned above and $M_{l}=M_{l}\left(q_{0}, p_{0}, k_{0}\right.$, $\left.\delta, s_{0}, M_{1}, M_{2}\right), l=6,7$. Moreover, (2.1) having $j=1$ can be written in the form

$$
\begin{gathered}
w_{1 \bar{z}}-\operatorname{Re}\left[Q_{11} w_{1 z}+A_{11} w_{1}\right]=A_{1}, \\
A_{1}=\operatorname{Re}\left[Q_{12} w_{2 z}+A_{12} w_{2}+A_{13} u_{1}+A_{14} u_{2}+A_{15}\right],
\end{gathered}
$$

and it is not difficult to see that no matter how large the constants $M_{1}$ and $M_{2}$ in (3.1) are, provided that the constants $q_{10}^{\prime}, k_{1 l}(l=2,3,4)$ in Condition $\mathrm{C}$ and $s_{1}$ in (1.10) are sufficiently small, we must have

$$
\begin{aligned}
& \left\|R S A_{1}\right\|_{L_{p_{0}}(\bar{G})} \leq s_{2}=s_{2}\left(q_{0}, p_{0}, k_{0}, \delta, s_{0}\right), \\
& C_{\alpha}\left[\left|z-t_{l}\right|^{\beta_{j l}}\left|z-t_{l-1}\right|^{\beta_{j l-1}}\left(\sigma_{11}(t) u_{1}(t)+\sigma_{12}(t) u_{2}(t)\right), \hat{\Gamma}_{l}\right] \\
& \leq s_{3}=s_{3}\left(q_{0}, p_{0}, k_{0}, \delta, s_{0}\right), l=1, \ldots, m .
\end{aligned}
$$

Noting that the complex equation (3.4) satisfies Condition $\mathrm{C}$ and $w_{1}(z)$ is a solution of the complex equation (3.4) with the boundary conditions (2.2)(2.6) $(j=1)$, we conclude that $w_{1}(z)$ satisfies the estimates:

$$
C_{\alpha}\left[R(z) w_{1}(z), \bar{G}\right] \leq M_{8},\left\|R S\left(\left|w_{1 \bar{z}}\right|+\left|w_{1 z}\right|\right)\right\|_{L_{p_{0}}(\bar{G})} \leq M_{9}
$$


where $M_{l}=M_{l}\left(q_{0}, p_{0}, k_{0}, \delta, s_{0}\right), l=8,9$. Substituting the function $w_{1}(z)$ into (2.6) with $j=1$, we have

$$
\begin{gathered}
C_{\delta}\left[R^{\prime}(z) u_{1}(z), \bar{G}\right] \leq M_{10}, C_{\delta}\left[R(z) w_{1}, \bar{G}\right], \\
\left\|R S\left(\left|u_{1 \overline{z z}}\right|+\left|u_{1 z z}\right|\right)\right\|_{L_{p_{0}}(\bar{G})} \leq M_{11},
\end{gathered}
$$

where $M_{l}=M_{l}\left(q_{0}, p_{0}, k_{0}, \delta, s_{0}\right), l=10,11$. Afterwards, we discuss the complex equation (2.1) with $j=2$, and rewrite it into the form

$$
\begin{gathered}
w_{2 \bar{z}}-\operatorname{Re}\left[Q_{22} w_{2 z}+A_{22} w_{2}\right]=A_{2}, \\
A_{2}=\operatorname{Re}\left[Q_{21} w_{1 z}+A_{21} w_{1}+A_{23} u_{1}+A_{24} u_{2}+A_{25}\right],
\end{gathered}
$$

On account of (3.1), (3.7), (3.8) and that $k_{24}$ and $s_{1}$ are sufficiently small, we have then

$$
\begin{aligned}
& \left\|R S A_{2}\right\|_{L_{p_{0}}(\bar{G})} \leq s_{4}=s_{4}\left(q_{0}, p_{0}, k_{0}, \delta, s_{0}\right), \\
& C_{\alpha}\left[\left|z-t_{l}\right|^{\beta_{j l}}\left|z-t_{l-1}\right|^{\beta_{j l-1}}\left(\sigma_{21}(t) u_{1}(t)+\sigma_{22}(t) u_{2}(t)\right), \Gamma_{l}\right] \\
& \leq s_{5}=s_{5}\left(q_{0}, p_{0}, k_{0}, \delta, s_{0}\right), l=1, \ldots, m .
\end{aligned}
$$

Because $u_{2}(z)$ and $w_{2}(z)$ satisfy $(2.2)-(2.6)$ with $j=2$, we can obtain the estimations

$$
\begin{aligned}
& C_{\delta}\left[R(z) w_{2}(z), \bar{G}\right] \leq M_{12},\left\|R S\left(\left|w_{2 \bar{z}}\right|+\left|w_{2 z}\right|\right)\right\|_{L_{p_{0}}(\bar{G})} \leq M_{13}, \\
& C_{\delta}\left[R^{\prime}(z) u_{2}(z), \bar{G}\right] \leq M_{14},\left\|R S\left(\left|u_{2 z \bar{z}}\right|+\left|u_{2 z z}\right|\right)\right\|_{L_{p_{0}}(\bar{G})} \leq M_{15},
\end{aligned}
$$

where $M_{l}=M_{l}\left(q_{0}, p_{0}, k_{0}, \delta, s_{0}\right), l=12, \ldots, 15$. From (3.7), (3.8), (3.12) and (3.13), we can derive (3.2). Moreover we may select $M_{1}=M_{3}$ and $M_{2}=M_{4}$ in (3.1).

Next, we consider the case $K_{1}<0$ or $K_{2}<0$. When $K_{2} \geq 0$, by the same reason as above, we have the estimates in (3.3). When $K_{2}<0$, in order to obtain (3.3), we use the estimates of the constants $h_{2 l}(l=[1-$ $\left.\left.(-1)^{2 K_{2}}\right] / 2, \ldots, N\right), h_{2 m}^{+}, h_{2 m}^{-}\left(m=1, \ldots,\left[\mid K_{2}+1 / 2\right]-1\right)$ determined by $w_{2}(z)$ satisfying

$$
\left|h_{2 l}\right|,\left|h_{2 m}^{+}\right|,\left|h_{2 m}^{-}\right| \leq s_{6}=s_{6}\left(q_{0}, p_{0}, k_{0}, \delta, s_{0}, M_{1}, M_{2}\right),
$$

and hence (3.3) is derived. When $K_{1}<0$, since $w_{1}(z)$ is a solution of the modified Riemann-Hilbert boundary value problem for (3.4) with the boundary condition (2.2)- $(2.6)(j=1)$, then similarly to the above, we can also prove that the constants $h_{1 l}\left(l=\left[1-(-1)^{2 K_{1}}\right] / 2, \ldots, N\right), h+_{1 m}, h_{1 m}^{-}\left(m=1, \ldots,\left[\mid K_{1}+\right.\right.$ $1 / 2]-1)$ satisfy the inequality (3.14) with $j=1$. It follows that (3.7) holds. The remanent part of the proof is the same as the case $K_{j} \geq 0(j=1,2)$. This completes the proof. 


\section{Solvability of Modified Problem and Original Problem for Nonlinear Systems of Elliptic Equations}

Suppose that the system (2.1) satisfies the following conditions, i.e. for any real functions $u_{j}^{n}(z)\left(R^{\prime}(z) u_{j}^{n}(z) \in C_{\delta}(\bar{G}), j, n=1,2\right)$, any complex functions $w_{j}^{n}(z)\left(R(z) w_{j}^{n}(z) \in C_{\delta}(\bar{G}), j, n=1,2\right), U_{j}(z)\left(R S U_{j} \in L_{p_{0}}(G), j=1,2\right)$ satisfy

$$
\begin{gathered}
F_{j}\left(z, u_{1}^{1}, u_{2}^{1}, w_{1}^{1}, w_{2}^{1}, U_{1}, U_{2}\right)-F_{j}\left(z, u_{1}^{2}, u_{2}^{2}, w_{1}^{2}, w_{2}^{2}, U_{1}, U_{2}\right)=\operatorname{Re}\left[\tilde{Q}_{j 1} U_{1}\right. \\
\left.+\tilde{Q}_{j 2} U_{2}+\tilde{A}_{j 1}\left(w_{1}^{1}-w_{1}^{2}\right)+\tilde{A}_{j 2}\left(w_{2}^{1}-w_{2}^{2}\right)+\tilde{A}_{j 3}\left(u_{1}^{1}-u_{1}^{2}\right)+\tilde{A}_{j 4}\left(u_{2}^{1}-u_{2}^{2}\right)\right], \\
j=1,2,
\end{gathered}
$$

where $\tilde{Q}_{j l}=\tilde{Q}_{j l}\left(z, u_{1}^{1}, u_{1}^{2}, u_{2}^{1}, u_{2}^{2}, w_{1}^{1}, w_{1}^{2}, w_{2}^{1}, w_{2}^{2}, U_{1}, U_{2}\right)(j, l=1,2) \tilde{A}_{j l}=\tilde{A}_{j l}\left(z, u_{1}^{1}\right.$, $\left.u_{1}^{2}, u_{2}^{1}, u_{2}^{2}, w_{1}^{1}, w_{1}^{2}, w_{2}^{1}, w_{2}^{2}, U_{1}, U_{2}\right)(j=1,2, l=1, \ldots, 4)$ satisfy the conditions

$$
\begin{aligned}
& \left|\tilde{Q}_{j 1}\right| \leq q_{j 0},\left|\tilde{Q}_{j 2}\right| \leq q_{j 0}^{\prime}\left(q_{j 0}+q_{j 0}^{\prime}<1\right), \\
& \left.|| A_{j l}\right|_{L_{p}(\bar{G})} \leq k_{j l}<k_{0}, j=1,2, l=1, \ldots, 4 .
\end{aligned}
$$

We first assume that the coefficients $Q_{j l}, A_{j l}$ of the first order system (2.1) equal zero in the neighborhood $\bar{G} \backslash G_{m}$ of the boundary $\Gamma$, where $G_{m}=\{z|| z-Z \mid \geq$ $1 / m, z \in \bar{G}, Z=\left\{t_{1}, \ldots, t_{m}\right\}, m$ is a sufficiently large positive integer, and write this system in the form

$$
w_{j \bar{z}}=f_{j}^{(m)}\left(z, u_{1}, u_{2}, w_{1}, w_{2}, w_{1 z}, w_{2 z}\right), j=1,2 .
$$

Theorem 4.1. Suppose that the system (4.2) of first order complex equations satisfies Condition $\mathrm{C}$ and (4.1). If the constants $q_{10}^{\prime}, k_{1 l}(l=2,3,4), k_{24}$ in (1.3), (1.4) and $s_{1}$ in the (1.10) are sufficiently small, then Problem Q for the system (4.2) is solvable.

Proof. Let us introduce the Banach space $B=C^{1}\left(G_{m}\right) \times C^{1}\left(G_{m}\right) \times L_{p_{0}}\left(G_{m}\right) \times$ $L_{p_{0}}\left(G_{m}\right)$ and denote by $B_{M}$ the totality of the systems of the functions: $\omega=$ $\left(u_{1}(z), u_{2}(z), \rho_{1}(z), \rho_{2}(z)\right)$ satisfying the inequalities

$$
C^{1}\left[u_{j}(z), G_{m}\right] \leq M_{3},\left\|\rho_{j}(z)\right\|_{L_{0}\left(G_{m}\right)} \leq M_{4}, j=1,2,
$$

where $M_{3}$ and $M_{4}$ are the constants similar to (3.2). Evidently, $B_{M}$ is a bounded open set in $B$. We arbitrarily select $\omega=\left(u_{1}(z), u_{2}(z), \rho_{1}(z), \rho_{2}(z)\right) \in \overline{B_{M}}$ and make the double integrals

$$
H \rho_{j}=\frac{2}{\pi} \iint_{G_{m}} \ln \left|1-\frac{z}{\zeta}\right| \cdot \rho_{j}(\zeta) \mathrm{d} \sigma_{\zeta}, j=1,2,
$$


and so

$$
\left(H \rho_{j}\right)_{z}=-\frac{1}{\pi} \iint_{G_{m}} \frac{\rho_{j}(\zeta)}{\zeta-z} \mathrm{~d} \sigma_{\zeta}=T \rho_{j}, j=1,2 .
$$

Substitute $u_{j}(z)$ and $T \rho_{j}(j=1,2)$ into suitable positions of the boundary conditions (2.2)-(2.6), and similarly to [3],[10], we can prove the existence and uniqueness of the analytic function $\Phi_{j}(z)$ in $G$ satisfying the boundary conditions

$$
\begin{gathered}
\operatorname{Re}\left[\overline{\lambda_{j}(t)}\left(\Phi_{j}(t)+T \rho_{j}\right)\right]+\sigma_{j 1}(t) u_{1}(t)+\sigma_{j 2}(t) u_{2}(t) \\
=\tau_{j 3}(t)+h_{j}(t) \overline{\lambda_{j}(z)} X_{j}(z), t \in \Gamma, \\
\operatorname{Im}\left[\overline{\lambda_{j}\left(a_{l}\right)}\left(\Phi_{j}(t)+T \rho_{j}\right)\right]_{t=a_{l}}=b_{j l}, l=J_{j}, K_{j} \geq 0, j=1,2,
\end{gathered}
$$

where $\Phi_{j}(z), w_{j}(z)=\Phi_{j}(z)+T \rho_{j}(j=1,2)$ satisfy the estimations

$$
C_{\delta}\left[\Phi_{j}^{\prime}(z), G_{m}\right] \leq M_{16}, C_{\delta}\left[w_{j}(z), G_{m}\right] \leq M_{17}, j=1,2,
$$

in which $M_{l}=M_{l}\left(q_{0}, p_{0}, k_{0}, \delta, s_{0}, G_{m}, M_{3}, M_{4}\right), l=16,17$. Now we substitute $u_{j}(z), w_{j}(z), \Phi_{j}^{\prime}(z)$ into the appropriate places of the system (4.2) and consider the system of the integral equations with the parameter $t(0 \leq t \leq 1)$ :

$$
\rho_{j}^{*}(z)=t f_{j}^{(m)}\left(z, u_{1}, u_{2}, w_{1}, w_{2}, \Phi_{1}^{\prime}(t)+\Pi \rho_{1}^{*}, \Phi_{2}^{\prime}(t)+\Pi \rho_{2}^{*}\right), j=1,2,
$$

where

$$
\Pi \rho_{j}^{*}=-\frac{1}{\pi} \iint_{G_{m}} \frac{\rho_{j}^{*}(\zeta)}{(\zeta-z)^{2}} \mathrm{~d} \sigma_{\zeta}, j=1,2 .
$$

By Condition $\mathrm{C}$ and the principle of contraction, the system (4.7) has the unique solutions $\rho_{j}^{*}(z) \in L_{p_{0}}\left(G_{m}\right), j=1,2$. Afterwards we seek the analytic functions $\Phi_{j}^{*}(z)(j=1,2)$ in $G$, which satisfy the boundary conditions:

$$
\begin{gathered}
\operatorname{Re}\left[\overline{\lambda_{j}(t)}\left(\Phi_{j}^{*}(t)+T \rho_{j}^{*}\right)\right]+\sigma_{j 1}(t) u_{1}(t)+\sigma_{j 2}(t) u_{2}(t) \\
=\tau_{j 3}(t)+h_{j}(t) \overline{\lambda_{j}(z)} X_{j}(z), \in \Gamma, \\
\operatorname{Im}\left[\overline{\lambda_{j}(t)}\left(\Phi_{j}^{*}(t)+T \rho_{j}^{*}\right)\right]_{t=a_{l}}=b_{j l}, \\
l \in J_{j}, K_{j} \geq 0, j=1,2,
\end{gathered}
$$

and then find single-value functions

$$
u_{j}^{*}(z)=b_{j 0}+\operatorname{Re} \int_{a_{0}}^{z}\left[w_{j}^{*}(z)+\sum_{k=1}^{N} \frac{i d_{j k}}{z-z_{k}} \mathrm{~d} z\right], j=1,2,
$$


where $w_{j}^{*}(z)=\Phi_{j}^{*}(z)+T \rho_{j}^{*}, j=1,2$. Denote by $\omega^{*}=T[\omega, t)(0 \leq t \leq 1)$ a mapping from $\omega=\omega(z)$ to $\omega^{*}=\left[u_{1}^{*}(z), u_{2}^{*}(z), \rho_{1}^{*}(z), \rho_{2}^{*}(z)\right]$. Moreover, if the constants $q_{10}^{\prime}, k_{1 l}(l=2,3,4), k_{24}$ in (1.3),(1.4) and $s_{1}$ in the boundary condition (1.10) are sufficiently small, then similarly to the proof of Theorem 3.1, we can derive that the solution $\omega=\left(u_{1}(z), u_{2}(z), \rho_{1}(z), \rho_{2}(z)\right) \in \overline{B_{M}}$ of Problem Q for the system

$$
\begin{gathered}
\rho_{j}(z)=t f_{j}^{(m)}\left(z, u_{1}, u_{2}, w_{1}, w_{2}, \Phi_{1}^{\prime}(t)+\Pi \rho_{1}, \Phi_{2}^{\prime}(t)+\Pi \rho_{2}\right), \\
0 \leq t \leq 1, j=1,2
\end{gathered}
$$

satisfies (4.2), where $\alpha, p_{0}$ are stated before and $M_{k}=M_{k}\left(q_{0}, p_{0}, k_{0}, G_{m}, \nu\right.$, $\left.s_{0}\right)(k=3,4)$, and hence $\omega=\left(u_{1}(z), u_{2}(z), \rho_{1}(z), \rho_{2}(z)\right) \in \overline{B_{M}}$. Further, we can verify that the mapping $\omega^{*}=T[\omega, t)(0 \leq t \leq 1)$ satisfies three conditions of the Leray-Schauder theorem, i.e.

1) In order to prove the complete continuity of $\omega^{*}=T[\omega, t)(0 \leq t \leq 1)$ in $\overline{B_{m}}$, we chose arbitrarily $\omega_{n} \in \overline{B_{M}}, n=1,2, \ldots$, and so $\omega_{n}^{*}=T\left[\omega_{n}, t\right]=$ $\left(u_{1 n}^{*}(z), u_{2 n}^{*}(z), \rho_{1 n}^{*}(z), \rho_{2 n}^{*}(z)\right) \in B$, i.e.

$$
\rho_{j n}^{*}(z)=t f_{j}^{(m)}\left(z, u_{1 n}, u_{2 n}, w_{1 n}, w_{2 n}, \Phi_{1 n}^{\prime}(t)+\Pi \rho_{1 n}^{*}, \Phi_{2 n}^{\prime}(t)+\Pi \rho_{2 n}^{*}\right), j=1,2 .
$$

We can prove as above that $w_{j n}(z)$ and $\Phi_{j n}^{\prime}(z)$ satisfy the estimation (4.6). Thus we may select subsequences of $\left\{u_{j n}(z)\right\},\left\{w_{j n}(z)\right\},\left\{\Phi_{j n}^{\prime}(z)\right\}$, which uniformly converge to $u_{j 0}(z), w_{j 0}(z), \Phi_{j 0}^{\prime}(z)$ in $G_{m}$ respectively, and denote by the same symbols the subsequences. Note that the right side of the system

$$
\rho_{j 0}^{*}(z)=t f_{j}^{(m)}\left(z, u_{10}, u_{20}, w_{10}, w_{20}, \Phi_{10}^{\prime}(t)+\Pi \rho_{10}^{*}, \Phi_{20}^{\prime}(t)+\Pi \rho_{20}^{*}\right), j=1,2 .
$$

equals zero for $z \notin G_{m}$, and apply the principle of contraction, then the system (4.13) has a unique solution $\left(\rho_{10}^{*}(z), \rho_{20}^{*}(z)\right)$ and $\rho_{j 0}^{*}(z) \in L_{p_{0}}\left(G_{m}\right), j=1,2$. Let (4.2) be minus (4.13), we have

$$
\begin{aligned}
& \rho_{j n}^{*}(z)-\rho_{j 0}^{*}(z)=t\left[f_{j}^{(m)}\left(z, u_{1 n}, u_{2 n}, w_{1 n}, w_{2 n}, \Phi_{1 n}^{\prime}(t)+\Pi \rho_{1 n}^{*}, \Phi_{2 n}^{\prime}(t)+\Pi \rho_{2 n}^{*}\right)\right. \\
& \left.-f_{j}^{(m)}\left(z, u_{1 n}, u_{2 n}, w_{1 n}, w_{2 n}, \Phi_{1 n}^{\prime}(t)+\Pi \rho_{10}^{*}, \Phi_{2 n}^{\prime}(t)+\Pi \rho_{20}^{*}\right)+c_{j n}(z)\right], \\
& c_{j n}(z)=f_{j}^{(m)}\left(z, u_{1 n}, u_{2 n}, w_{1 n}, w_{2 n}, \Phi_{1 n}^{\prime}(t)+\Pi \rho_{10}^{*}, \Phi_{2 n}^{\prime}(t)+\Pi \rho_{20}^{*}\right) \\
& -f_{j}^{(m)}\left(z, u_{10}, u_{20}, w_{10}, w_{20}, \Phi_{10}^{\prime}(t)+\Pi \rho_{10}^{*}, \Phi_{20}^{\prime}(t)+\Pi \rho_{20}^{*}, j=1,2 .\right.
\end{aligned}
$$


By virtue of Condition $\mathrm{C}$, we can derive $\left\|c_{j n}(z)\right\|_{L_{p_{0}}\left(G_{m}\right)} \rightarrow 0, j=1,2$, when $n \rightarrow \infty$, and from (4.14), it follows that

$$
\begin{gathered}
\sum_{j=1}^{2}\left\|c_{j n}^{*}(z)-c_{j 0}^{*}(z)\right\|_{L_{p_{0}}\left(G_{m}\right)} \\
\leq \frac{1}{1-2 \max _{j=1,2}\left(q_{j 0}+q_{j 0}^{\prime}\right) \| \Pi||_{L_{p_{0}}}} \sum_{j=1}^{2}\left\|c_{j n}\right\|_{L_{p_{0}}\left(G_{m}\right)} .
\end{gathered}
$$

Therefore, $\sum_{j=1}^{2}\left\|c_{j n}^{*}(z)-c_{j 0}^{*}(z)\right\|_{L_{p_{0}}\left(G_{m}\right)} \rightarrow 0$ for $n \rightarrow \infty$. Besides, according to $C\left[w_{j n}^{*}(z)-w_{j 0}^{*}(z), G_{m}\right] \rightarrow 0$ for $n \rightarrow \infty$, it is clear that $C^{1}\left[u *_{j n}(z)-\right.$ $\left.u_{j 0}^{*}(z), G_{m}\right] \rightarrow 0(j=1,2)$ as $n \rightarrow \infty$. This shows the complete continuity of $\omega^{*}=T[\omega, t](0 \leq t \leq 1)$ in $\overline{B_{m}}$. By the same reason, we can also prove that $\omega^{*}=T[\omega, t](0 \leq t \leq 1)$ maps continuously $\overline{B_{m}}$ onto $B$, and $T[\omega, t)$ is uniformly continuous with respect to $t \in[0,1]$ for $\omega \in \overline{B_{M}}$.

2) When $t=0$, it is easy to see that $\omega^{*}=T[\omega, 0]=\left(u_{1}^{*}(z), u_{2}^{*}(z), 0,0\right) \in B_{M}$ from (4.7) and (4.10).

3) From (3.2), we find that $\omega=T[\omega, t](0 \leq t \leq 1)$ has not a solution $\omega(z)$ on the boundary $\partial B_{M}=\overline{B_{M}} \backslash B_{M}$.

Hence by the Leray-Schauder theorem, we can know that the system (4.11) with $t=1$ has a solution $\omega=\left(u_{1}(z), u_{2}(z), \rho_{1}(z), \rho_{2}(z)\right) \in B_{M}$, and thus $\left(u_{1}(z), u_{2}(z), w_{1}(z), w_{2}(z)\right)$ is a solution of Problem Q for the first order system (4.1), where $w_{j}(z)=\Phi_{j}(z)+T \rho_{j}(j=1,2)$.

Theorem 4.2. Let the first order system (2.1) of the complex equations satisfy Condition $\mathrm{C}$ and (4.1). If the constants $q_{10}^{\prime}, k_{1 l}(l=2,3,4), k_{24}$ in (1.3), (1.4) and $s_{1}$ in (1.10) are sufficiently small, then Problem Q for the system (2.1) is solvable.

Proof. On the basis of Theorems 4.1 and 3.1, it is easy to see that Problem $\mathrm{Q}$ for the system $(4.2)(m=1,2, \ldots)$ have solutions $u_{j m}(z), w_{j m}(z)=$ $\Phi_{j m}(z)+T \rho_{j m}(j=1,2, m=1,2, \ldots)$, and these solutions satisfy the estimation (3.2). Hence we can choose subsequences $\left\{u_{j m}^{*}(z)\right\},\left\{w_{j m}^{*}(z)\right\}$ of $\left\{u_{j m}(z)\right\}$, $\left\{w_{j m}(z)\right\}$, which converge to $u_{j 0}(z), w_{j 0}(z)(j=1,2)$ in $G^{*}$ respectively, and $u_{j 0}(z), w_{j 0}(z)(j=1,2)$ satisfy the boundary conditions $(2.2)-(2.5)$ and the relation (2.6).

In the following, we shall prove that $\left(u_{10}(z), u_{20}(z), w_{10}(z), w_{20}(z)\right)$ is a solution of the first order system (2.1). Because $u_{j m}(z), w_{j m}(z)=\Phi_{j m}(z)+$ $T \rho_{j m}(j=1,2)$ satisfy $(3.1)$, we may choose the subsequences $\left\{\rho_{j m}^{*}(z)\right\}$ of $\left\{\rho_{j m}(z)\right\}=\left\{w_{j m \bar{z}}\right\}(j=1,2)$, which weakly converges to $\rho_{j 0}(z) \in L_{p_{0}}\left(G_{n}\right)(j=$ 
$1,2)$ for any fixed large positive integer $n$. Therefore $\left\{\Phi_{j m}^{*}(z)\right\}$ and $\left\{T \rho_{j m}^{*}\right\}$ uniformly converge to $\Phi_{j 0}(z), T \rho_{j 0}(j=1,2)$ in $G_{n}$ respectively, where $\Phi_{j 0}(z)(j=$ $1,2)$ are analytic functions, and $w_{j 0}(z)=\Phi_{j 0}(z)+T \rho_{j 0} \in W_{p_{0}}^{1}\left(G_{n}\right)(j=1,2)$, it is clear that $G_{n}$ is any compact subset of $G^{*}$. For convenience, we denote still $\left\{u_{j m}^{*}(z)\right\},\left\{w_{j m}^{*}(z)\right\},\left\{\rho_{j m}^{*}(z)\right\}$ by $\left\{u_{j m}(z)\right\},\left\{w_{j m}(z)\right\},\left\{\rho_{j m}(z)\right\}$ respectively. In particular, let $S=\left\{z|| z-z_{*} \mid \leq R,\left\{\left|z-z_{*}\right|=R\right\} \cap Z=\emptyset\right\}\left(z_{*} \in G^{*}, R>0\right)$ be a set in $G^{*}$, and $w_{j m}(z)(m=1,2, \ldots)$ admit the expression in $S$ :

$$
\begin{gathered}
w_{j m}(z)=\Psi_{j m}(z)+P \rho_{j m}, P \rho_{j m}=Q \rho_{j m}-\left.\operatorname{Im} Q \rho_{j m}\right|_{z=z_{*}}, \\
Q \rho_{j m}=-\frac{1}{\pi} \iint_{S}\left[\frac{\rho_{j m}(\zeta)}{\zeta-z}+\frac{\left(z-z_{*}\right) \rho_{j m}(\zeta)}{R^{2}-(\bar{\zeta}-\bar{z})\left(z-z_{*}\right)}\right] \mathrm{d} \sigma_{\zeta}, \\
j=1,2, m=1,2, \ldots
\end{gathered}
$$

We note that $\left\{\rho_{j m}(z)\right\}$ weakly converge to $\rho_{j 0}(z)(j=1,2)$ in $S,\left\{\Psi_{j m}^{\prime}(z)\right\}$ uniformly converge to $\Psi_{j 0}^{\prime}(z)$ in $S$ and $\Psi_{j m}^{\prime}(z)$ satisfy

$$
\left\|\Psi_{j m}^{\prime}(z)\right\|_{L_{p_{0}}(S)} \leq M_{18}=M_{18}\left(q_{0}, p_{0}, k_{0}, \delta, S, s_{0}\right), j=1,2, m=1,2, \ldots,
$$

and apply the principle of contraction that the system of the integral equations

$$
\rho_{j 0}^{*}(z)=f_{j}\left(z, u_{10}, u_{20}, w_{10}, w_{20}, \Psi_{10}^{\prime}+Q \rho_{10}^{*}, \Psi_{20}^{\prime}(t)+Q \rho_{20}^{*}\right), j=1,2,
$$

has a unique solution $\rho_{j 0}^{*}(z) \in L_{p_{0}}(S)$, where $Q \rho_{j 0}^{*}=\left(P \rho_{j 0}^{*}\right)_{z}, j=1,2$. Let the system

$$
\begin{gathered}
\rho_{j m}^{*}(z)=f_{j}^{(m)}\left(z, u_{1 m}, u_{2 m}, w_{1 m}, w_{2 m}, \Psi_{1 m}^{\prime}+Q \rho_{1 m}^{*}, \Psi_{2 m}^{\prime}(t)+Q \rho_{2 m}^{*}\right), \\
j=1,2, m=1,2, \ldots
\end{gathered}
$$

be minus (4.13), and notice that $f_{j}=f_{j}^{(m)}$ in $S, j=1,2$, when $m$ ia large enough. We can prove that $\sum_{j=1}^{2}\left\|\rho_{j m}^{*}(z)-\rho_{j 0}^{*}(z)\right\|_{L_{p_{0}}(S)} \rightarrow 0$ for $m \rightarrow \infty$. Hence $\left(u_{10}(z), u_{20}(z), w_{10}(z), w_{20}(z)\right)$ is a solution of $(2.1)$ in $S$, where $w_{j 0}(z)=$ $\Psi_{j 0}(z)+P \rho_{j 0}^{*}=\Psi_{j 0}(z)+P \rho_{j 0}(j=1,2)$. Owing to the arbitrariness of $S$, $\left(u_{10}(z), u_{20}(z), w_{10}(z), w_{20}(z)\right)$ is therefore a solution of the system (2.1) in $G$. This completes the proof.

In the following, we shall derive the results of solvability on Problem $\mathrm{P}$ of the second order system (1.1).

Proof of Theorem 1.1. From Theorem 4.2, we know that Problem Q of the first order system $(2.1)$ has a solution $\left(u_{1}(z), u_{2}(z), w_{1}(z), w_{2}(z)\right)$. Let us substitute the solution into the boundary conditions (2.2)-(2.4). The functions 
$h_{j}(t)(j=1,2)$ and the constants $d_{j k}(j=1,2, k=1, \ldots, N)$ are then determined. If the functions and the constants are equal zero, i.e.

$$
\begin{aligned}
& h_{j}(t)=\left\{\begin{array}{l}
h_{j l}=0, l=1, \ldots, N, \quad \text { when } K_{j} \geq 0, \\
h_{j l}=0, l=\left[1-(-1)^{2 K_{j}}\right] / 2, \ldots, N, \\
\eta_{j m}^{ \pm}=0, m=1, \ldots,\left[\left|K_{j}\right|+1 / 2\right]-1,
\end{array}\right\} \text { when } K_{j}<0, j=1,2, \\
& d_{j k}=0, j=1,2, k=1, \ldots, N,
\end{aligned}
$$

then the solution $\left(u_{1}(z), u_{2}(z), w_{1}(z), w_{2}(z)\right)$ of Problem $\mathrm{Q}$ for the first order system (2.1) is also a solution of Problem P for the second order system (1.1), and $u_{j z}=w_{j}(z), j=1,2$. From (4.20) and (4.21), it is not difficult to see the total number of solvability conditions of Problem $\mathrm{P}$ for the system (1.1). For example, when the indices $K_{j} \geq 0(j=1,2)$, Problem P for the system (1.1) has $4 N$ solvability conditions. Besides the solution of Problem $\mathrm{P}$ depends on some arbitrarily real constants. For instance, when $K_{j} \geq 0(j=1,2)$, the solution of Problem P depends on $2\left(K_{1}+K_{2}+2\right)$ arbitrarily real constants. As for the other cases, we use the same way to obtain also the results as stated in Theorem 1.1.

\section{Acknowledgments}

The research was supported by PHR (IHLB 201106206), and College Students Scientific Research and Undertaking Starting Action Project (SJ201301016).

\section{References}

[1] I.N. Vekua, Generalized Analytic Functions. Pergamon, Oxford (1962).

[2] A.V. Bitsadze, Some Classes of Partial Differential Equations, Gordon and Breach, New York (1988).

[3] G.C. Wen, Linear and Nonlinear Elliptic Complex Equations, Shanghai Scientific and Technical Publishers, Shanghai (1986) (Chinese).

[4] G.C. Wen, Conformal Mappings and Boundary Value Problems, Translations of Mathematics Monographs 106, Amer. Math. Soc., Providence, RI (1992).

[5] G.C. Wen, C.W., M.Y. Tian, Function Theoretic Methods of Free Boundary Problems and Their Applications to Mechanics, Higher Education Press, Beijing (1996) (Chinese). 
[6] G.C. Wen, Linear and Nonlinear Elliptic Complex Equations, Shanghai Scientific and Technical Publishers, Shanghai (1986) (Chinese).

[7] G.C. Wen, Approximate Methods and Numerical Analysis for Elliptic Complex Equations, Gordon and Breach, Amsterdam (1999).

[8] G.C. Wen, B.T. Zou, Initial-Boundary Value Problems for Nonlinear Parabolic Equations in Higher Dimensional Domains, Science Press, Beijing (2002).

[9] S. Huang, Y.Y. Qiao, G.C. Wen, Real and Complex Clifford Analysis, Springer Verlag, Heidelberg (2005).

[10] G.C. Wen, D.C. Chen, Z.L. Xu, Nonlinear Complex Analysis and its Applications, Mathematics Monograph Series 12, Science Press, Beijing (2008).

[11] G.C. Wen, Recent Progress in Theory and Applications of Modern Complex Analysis, Science Press, Beijing (2010).

[12] G.C. Wen, Linear and Quasilinear Complex Equations of Hyperbolic and Mixed Type, Taylor \& Francis, London (2002). 